\title{
Further Studies on the Origins of Asymmetric Charge Partitioning in Protein Homodimers
}

\author{
John C. Jurchen, David E. Garcia, and Evan R. Williams \\ Department of Chemistry, University of California at Berkeley, Berkeley, California, USA
}

\begin{abstract}
Dissociation of gas-phase protonated protein dimers into their constituent monomers can result in either symmetric or asymmetric charge partitioning. Dissociation of $\alpha$-lactalbumin homodimers with $15+$ charges results in a symmetric, but broad, distribution of protein monomers with charge states centered around $8+/ 7+$. In contrast, dissociation of the $15+$ heterodimer consisting of one molecule in the oxidized form and one in the reduced form results in highly asymmetric charge partitioning in which the reduced species carries away predominantly $11+$ charges, and the oxidized molecule carries away $4+$ charges. This result cannot be adequately explained by differential charging occurring either in solution or in the electrospray process, but appears to be best explained by the reduced species unfolding upon activation in the gas phase with subsequent separation and proton transfer to the unfolding species in the dissociation complex to minimize Coulomb repulsion. For dimers of cytochrome $c$ formed directly from solution, the $17+$ charge state undergoes symmetric charge partitioning whereas dissociation of the $13+$ is asymmetric. Reduction of the charge state of dimers with $17+$ charges to $13+$ via gas-phase proton transfer and subsequent dissociation of the mass selected $13+$ ions results in a symmetric charge partitioning. This result clearly shows that the structure of the dimer ions with 13+ charges depends on the method of ion formation and that the structural difference is responsible for the symmetric versus asymmetric charge partitioning observed. This indicates that the asymmetry observed when these ions are formed directly from solution must come about due either to differences in the monomer conformations in the dimer that exist in solution or that occur during the electrospray ionization process. These results provide additional evidence for the origin of charge asymmetry that occurs in the dissociation of multiply charged protein complexes and indicate that some solution-phase information can be obtained from these gas-phase dissociation experiments. (J Am Soc Mass Spectrom 2004, 15, 1408-1415) (c) 2004 American Society for Mass Spectrometry
\end{abstract}

$\mathrm{T}$ he biological functionality of proteins and protein complexes is highly dependent on their solutionphase structures. The importance of the field of structural biology is reflected in several recently awarded Nobel prizes for contributions to techniques for structural elucidation, including NMR (Kurt Wuthrich 2002 in chemistry), X-ray crystallography (Johann Deisenhofer, Robert Huber, and Hartmut Michel 1988 in chemistry; Herbert Hauptman and Jerome Karle 1985 in chemistry), and crystallographic electron microscopy (Sir Aaron Klug 1982 in chemistry). The analysis of proteins and protein complexes by these methods can provide detailed, three-dimensional information, but these methods are often limited by experimental constraints, such as the need to obtain useful protein crystals, or the requirement of substantial amounts of sample. In contrast, mass spectrometry requires little

Published online September 3, 2004

Address reprint requests to Dr. E. R. Williams, Department of Chemistry, University of California at Berkeley, Berkeley, CA 94720, USA. E-mail: williams@cchem.berkeley.edu sample, and with electrospray ionization (ESI) or soft laser desorption/ionization (John Fenn and Kiochi Tanaka 2002 in chemistry), a wide variety of biomolecules and specific complexes can be introduced into a mass spectrometer. A number of methods have been developed to probe the general three-dimensional shapes of biomolecule ions and noncovalent complexes using the techniques of ion mobility [1-8], H/D exchange both in solution $[9,10]$ and the gas phase [11-28], and proton-transfer reactivity [29, 30].

For the analysis of noncovalent complexes, mass spectrometry has the advantage that the stoichiometry of the complex can be determined from a simple mass measurement [31]. In some cases, MS can provide the correct solution-phase binding constants for proteinligand interactions [32-35]. These developments have been reviewed in detail [31, 35]. In principle, information about the structure of noncovalent complexes can potentially be obtained by examining the product ions resulting from dissociation of the complex in a tandem mass spectrometry (MS/MS) experiment. The extent of relevant information that can be obtained from these 
experiments depends on the amount of solution-phase structural information that is retained by biological complexes upon transmission into the gas phase (or if this information can be reconstructed based on knowledge of structural changes that occur during this transition) and the degree to which MS/MS techniques disrupt any remaining structural information. Many experiments show that gas-phase biomolecule complexes and even some small molecules can retain a "memory" of their solution-phase structure and that this memory can be probed by dissociation processes [36-48].

A number of MS/MS studies of homogenous biological complexes (tetramers, pentamers, hexamers, etc.) have revealed the initially surprising result that the primary dissociation pathway of multimeric complexes is the ejection of a highly charged monomer that removes $30-50 \%$ of the charge of the complex [49-53]. $X$-ray crystallography indicates that the solution structures of the subunits in many homogeneous complexes are identical. In some cases, the entire complex crystallizes within the asymmetric unit of the crystal, as is the case with the Shiga-like toxin [9], while in others, such as Apostreptavidin, the asymmetric unit contains a single subunit, and the structure of the complex is extrapolated by molecular modeling [54]. Due to the virtually identical solution-phase structures of the participating subunits within these biological complexes, the observed asymmetric charge partitioning appears to be an artifact of the ESI-MS/MS procedure. It has been suggested that asymmetric charge partitioning correlates with gas-phase conformational rearrangements $[50,52,55,56]$. It has also been demonstrated that the degree of asymmetric charge partitioning for protein homodimers depends on the conformational flexibility of the proteins in the complex, the charge state of the complex, the internal energy deposition, and the composition of the electrospray solutions from which the complexes are formed [56]. For larger clusters consisting of identical subunits, fission was observed to occur with preferential formation of protonated dimers, and with increasing charge state and cluster size, increasing formation of protonated monomer was observed [57]. The extent of fission versus neutral loss for these clusters could be related to that observed for multiply charged metal clusters with one important difference: Differences in conformational flexibility of the monomer sub-units within a cluster results in differences in the branching ratios of the various dissociation processes observed for these multiply protonated biomolecule clusters [57].

Gas-phase hydrogen-deuterium (H/D) exchange is typically done by storing an ion of interest in vacuum and measuring the change in ion mass due to $H / D$ exchange reactions that occur during collisions with neutral molecules of an exchange reagent, such as $\mathrm{D}_{2} \mathrm{O}$ or $\mathrm{ND}_{3}$. Although gas-phase $\mathrm{H} / \mathrm{D}$ exchange has been used extensively to characterize peptides and small molecules [11-21], and the structure of some gas-phase proteins [22-24], very little work has been done with noncovalent complexes involving biological ions, and this noncovalent work has focused on peptide dimers $[25,26]$ and peptide complexes with metal ions $[16,18$, $21,27]$ and small molecules [15, 28].

Here, further characterization of protein homodimers using gas-phase dissociation and gas-phase H/D exchange is presented. Dissociation studies of mixed dimers of oxidized and reduced $\alpha$-lactalbumin provide further evidence that conformational flexibility plays a key role in determining the extent of asymmetric charge partitioning. It is shown for the dissociation of cytochrome $c$ homodimers that charge states prepared by gas-phase charge reduction from higher charge-state homodimers dissociate very differently than those prepared directly from solution. The first demonstration of gas-phase $H / D$ exchange of a protein complex is presented. These results indicate that two different mechanisms are responsible for the asymmetric charge partitioning observed in the dissociation of protein homodimers.

\section{Experimental}

Experiments were performed on the Berkeley-Bruker 9.4 T narrow bore Fourier-transform ion cyclotron resonance (FT-ICR) mass spectrometer that is described elsewhere [56]. The experimental procedures used are similar to those used previously [56]. Brief descriptions and additional details are described below.

\section{Chemicals}

Horse heart cytochrome $c, \alpha$-lactalbumin from bovine milk and triethylamine were purchased from SigmaAldrich Co. (St. Louis, MO), and were used without further purification. Deuterated ammonia was purchased from Cambridge Isotope Laboratories (Woburn, MA). Acetamidation of $\alpha$-lactalbumin was done by Dr. David King (University of California, Berkeley). All four intramolecular disulfide bonds of $\alpha$-lactalbumin are reduced with tris-(carboxyethyl)phosphine, acetamidated in the dark with iodoacetamide for one $h$ and purified using HPLC to remove buffer and unreacted reagents. Essentially complete reduction and acetamidation of all disulfide bonds in $\alpha$-lactalbumin were confirmed by mass measurements.

\section{Dimer formation}

Formation of non-specific protein dimers is enhanced by using protein concentrations of $100-500 \mu \mathrm{M}$ and adjusting interface voltages for "gentle" ion introduction. Dimer signal is improved by tuning for selective ion accumulation in the external hexapole in the source region of the instrument. This is done by accumulating the ions in the hexapole for 3-4 s (versus $\leq 1.0 \mathrm{~s}$ typically), increasing the hexapole DC offset from $\sim 2.7$ $\mathrm{V}$ to $3.5-4.5 \mathrm{~V}$, and injecting multiple hexapole accumulations into the ion cell prior to detection. 
Mixed dimers of oxidized (all four disulfide bonds intact) and reduced (all four disulfide bonds broken and all eight cysteine residues subsequently acetamidated) $\alpha$-lactalbumin were formed by mixing solutions of the two $\alpha$-lactalbumin forms in different ratios. The total protein concentration was kept constant at $\sim 200 \mu \mathrm{M}$ and the ratios of the two components were altered to maximize the absolute abundance of mixed dimer. The greatest abundance of mixed dimer appears at relative concentrations of reduced to oxidized $\alpha$-lactalbumin of $\sim 2: 1$. Because the protein concentrations were determined by the mass of the lyophilized powders used to produce the solution, these concentrations are only approximate. Protein dimer ions were formed using ESI with a flow rate of $\sim 1 \mu \mathrm{L} / \mathrm{min}$ with solution conditions of $1: 1$ water:methanol $+2 \%$ acetic acid.

Cytochrome $c$ dimers were formed using regular ESI at a flow rate of $1-2 \mu \mathrm{L} / \mathrm{min}$ and a concentration of 100 $\mu \mathrm{M}$ in 1:1 water:methanol $+2 \%$ acetic acid. Dimer charge states formed directly from solution range from $\left[(\text { cytochrome } c)_{2}+19 \mathrm{H}\right]^{19+}$ to $\left[(\text { cyctochrome } c)_{2}+\right.$ $13 \mathrm{H}]^{13+}$ (abbreviated D19-D13). Dimer charge states at higher $m / z$ than D17 were ejected from the ion cell using correlated shots leaving D19-D17 charge states remaining in the ion cell. The FT-ICR vacuum chamber was conditioned for charge reduction by briefly introducing triethylamine into the vacuum chamber at a pressure of $\sim 3 \times 10^{-8}$ torr using a piezoelectric pulsed valve. The pulsed valve was then closed after $5 \mathrm{~s}$ and the base pressure of the instrument returned to $\sim 8 \times 10^{-9}$ torr after $95 \mathrm{~s}$. Charge reduction via proton transfer was done by trapping the cytochrome $c$ homodimers of charge states D19-D17 in the instrument until collisions with residual triethylamine molecules reduced the charge state of the dimers over the course of $100 \mathrm{~s}$ such that the $13+$ was the most abundant form of the dimer. The newly produced D13 ions were isolated using correlated sweeps and dissociated with sustained off resonance irradiation collisionally-activated dissociation (SORI-CAD) [58] at $+600 \mathrm{~Hz}$ off resonance and 3.5 $\mathrm{V}$ pk-pk for $0.25 \mathrm{~s}$ after the application of a pulse of nitrogen that increased the pressure in the vacuum chamber to $\sim 10^{-6}$ torr.

\section{Gas-phase H/D Exchange of Protein Complexes}

Gas-phase H/D exchange of cytochrome $c$ homodimers was done by trapping all electrospray generated ions in the cell and introducing $\mathrm{ND}_{3}$ into the vacuum chamber using a manual leak valve (Varian, Lexington, MA) for $180 \mathrm{~s}$ at several pressures ranging from $\sim 1 \times 10^{-8}$ to a maximum of $\sim 3 \times 10^{-7}$ torr. After $180 \mathrm{~s}$ of exchange, the leak valve was closed, and the vacuum chamber was pumped out for 60-120 s until the base pressure was sufficiently low for isotopic resolution $\left(<1 \times 10^{-8}\right.$ torr). Previous gas-phase $\mathrm{H} / \mathrm{D}$ exchange experiments with proteins have used $\mathrm{D}_{2} \mathrm{O}$ as the exchange reagent [22-24, 59]. Due to difficulties experienced with long trapping times $(>300 \mathrm{~s})$, and the considerable time

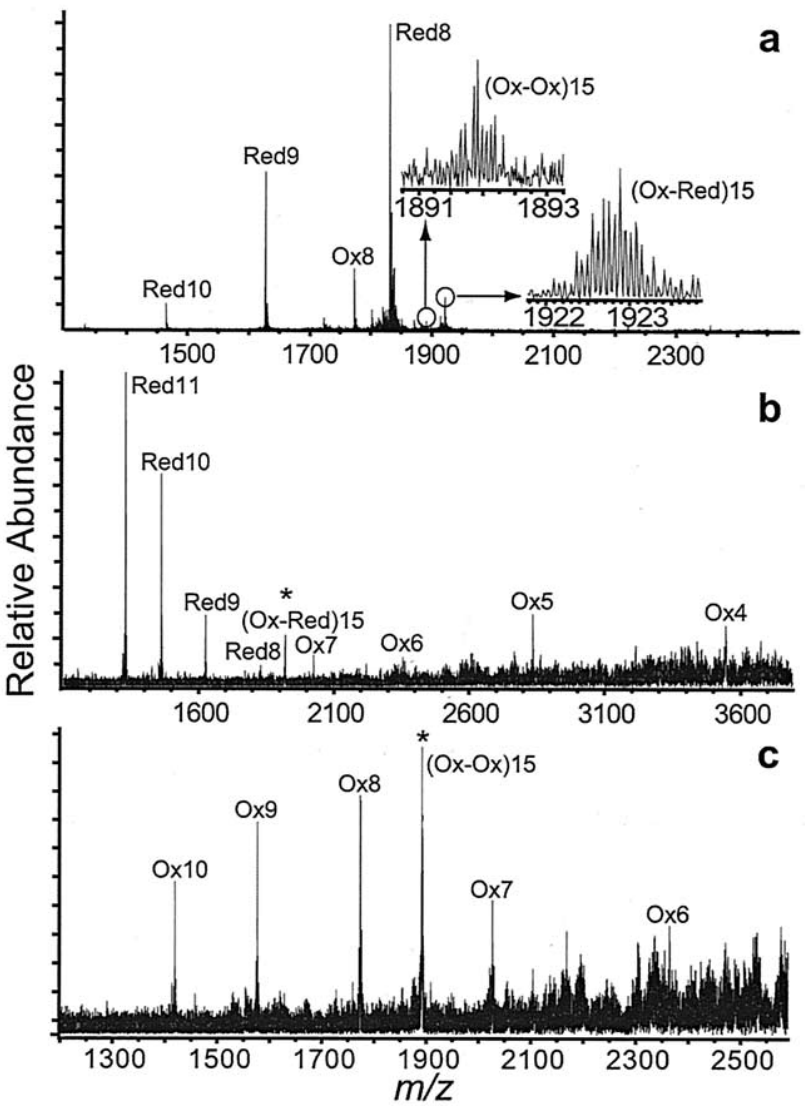

Figure 1. (a) ESI mass spectrum obtained from a $200 \mu \mathrm{M}$ solution (total protein concentration) containing oxidized (disulfide intact) and reduced $\alpha$-lactalbumin (1:1 water:methanol $+2 \%$ acetic acid), (b) MS/MS spectrum of the $\alpha$-lactalbumin oxidized-reduced hetero dimer with 15 protons, (Ox-Red)15, and (c) MS/MS spectrum of $\alpha$-lactalbumin oxidized-oxidized homo dimer, (Ox-Ox)15, formed from a solution of $\sim 125 \mu \mathrm{M}$ oxidized $\alpha$-lactalbumin in 1:1 water:methanol $+2 \%$ acetic acid. Red 8 represents (reduced $\alpha$-lactalbumin $+8 \mathrm{H})^{8+}$, etc.

required to remove the relatively nonvolatile $\mathrm{D}_{2} \mathrm{O}, \mathrm{ND}_{3}$ was chosen as the exchange reagent. The pressure of $\mathrm{ND}_{3}$ introduced into the vacuum chamber was measured using an uncalibrated ion gauge located $\sim 1.1 \mathrm{~m}$ from the ion cell.

\section{Results and Discussion}

\section{Dissociation of Mixed $\alpha$-Lactalbumin Dimers}

Using ESI solutions with high protein concentrations, it is possible to form both oxidized $\alpha$-lactalbumin protein homodimers (each protein has four intact disulfide bonds) and oxidized-reduced $\alpha$-lactalbumin heterodimers (one protein has four intact disulfide bonds and the other has four reduced disulfide bonds with all eight cysteine residues acetamidated). Both the oxidized homodimers and oxidized-reduced heterodimers form directly from ESI with 15 attached protons ((OxOx)15 and (Ox-Red)15, respectively) (Figure 1a). The (Ox-Ox)15 dissociates with a fairly broad, but symmetric charge partitioning (Figure 1c) similar to what has 
been observed previously for (Ox-Ox)13 and (RedRed)17 [56]. (Red-Red)13 was previously shown to dissociate asymmetrically to predominantly form M9/M4 [56]. In contrast to the (Ox-Ox)15, (Ox-Red)15 dissociates with highly asymmetric charge partitioning (Figure $1 b$ ). The most abundant dissociation process for the dimer dissociation is $(\mathrm{Ox}-\mathrm{Red}) 15 \rightarrow \operatorname{Red} 11+\mathrm{Ox} 4$, a partitioning process whereby the reduced $\alpha$-lactalbumin removes nearly $3 / 4$ of the total charge! Note that the greater signal intensity of the Red11 compared with that of the Ox4 is due to both the lower charge state of the latter (FT-ICR signal is proportional to charge) and the slightly lower detection efficiency at higher $\mathrm{m} / \mathrm{z}$.

The origin of the highly asymmetric charge partitioning could be due to asymmetric partitioning that occurs in the gas phase [56] or to asymmetry in the conformation or charge of the monomers that make up the dimer either in solution or induced by the electrospray processes. In the former mechanism, the asymmetric charge partitioning occurs because a conformational change of one of the monomer sub-units that makes up the dimer takes place upon activation. As the monomer unfolds, the molecule goes over the activation barrier for unfolding and separation. Because of the Coulomb energy, the exit of this barrier is highly repulsive and as one part of the unfolding molecule exits this channel, it will drive the unfolding of the rest of the molecule as it separates from the other monomer sub-unit. In this process, protons will transfer to the exiting unfolding ion to minimize Coulomb energy [30]. Because the reduced protein is more conformationally flexible and can unfold to a significantly greater extent than the oxidized protein, the reduced protein is the one that unfolds and carries away more charge than the oxidized form.

An alternative mechanism for this charge asymmetry is that the (Ox-Red) 15 could be formed in solution by the aggregation of highly charged reduced and lowly charged oxidized $\alpha$-lactalbumin monomers in solution, or formed during electrospray by asymmetric charging of the individual protein constituents during the final stages of ESI [60]. It has also been proposed that asymmetric charge partitioning for heterodimers may be related to differences in pI [61, 62]. Although a difference in charging for these two molecules occurs, it does not account for the magnitude of the asymmetric charge partitioning. In the ESI mass spectrum of mixed $\alpha$-lactalbumin solution, reduced $\alpha$-lactalbumin appears predominantly at $8+$ and to a lesser extent at $9+$ and $10+$ (Figure 1a). Very little 11+ is observed. Oxidized $\alpha$-lactalbumin occurs only at the $8+$ charge state. In the dissociation spectrum of (Ox-Red)15, Red11 and Ox5 are the dominant species observed, despite the fact that the abundances of these species produced directly by ESI is negligible. Although one could rationalize the lower charge state observed for Ox to the slightly lower charge state of the dimer versus the sum of the charge states of the individual species, this cannot hold true for the reduced species, which has a significantly higher charge in the dissociation spectrum than when formed

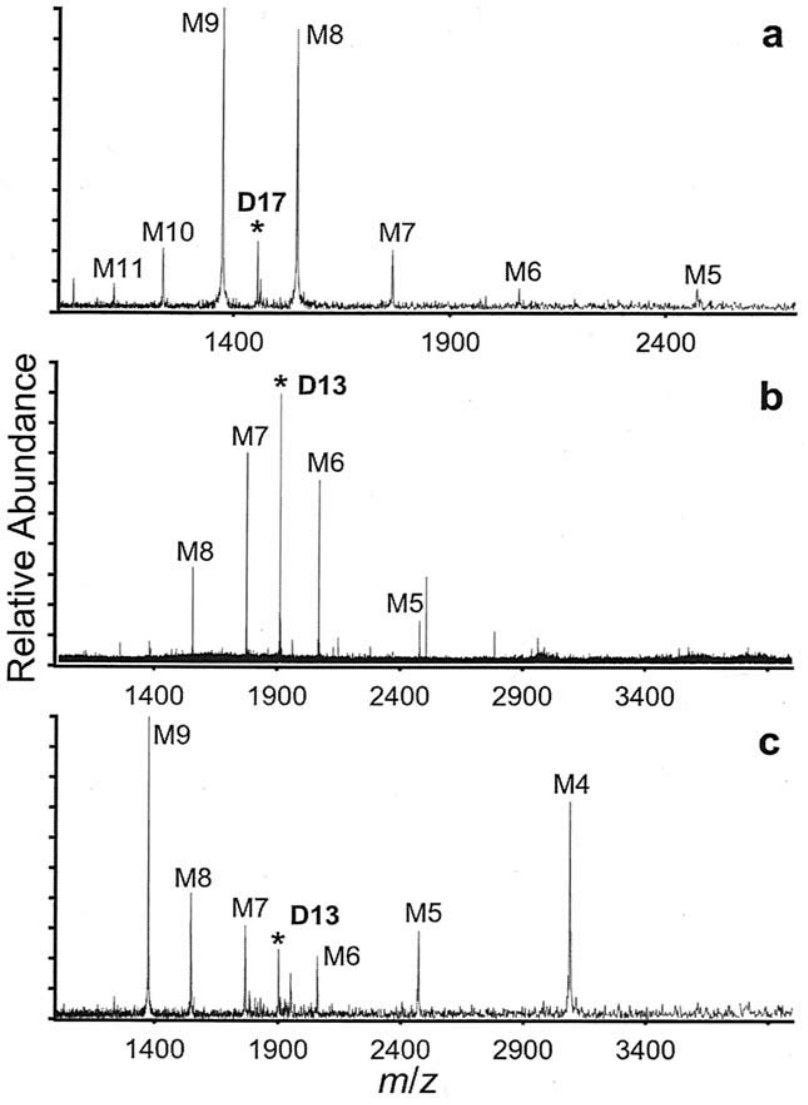

Figure 2. MS/MS spectra of (a) (cytochrome $c$ dimers $+17 \mathrm{H})^{17+}$, (D17), formed directly by ESI, (b) (cytochrome $c$ dimers + $13 \mathrm{H})^{13+}$, (D13), formed by isolation of D19-D17, gas-phase proton transfer reduction with diethylamine, isolation and subsequent activation of D13, and (c) D13 ions formed directly from solution. Ions were formed from a $100 \mathrm{mM}$ solution (1:1 water:methanol + $2 \%$ acetic acid).

directly by electrospray. Thus, this result is difficult to rationalize based solely on a model in which the asymmetric charge partitioning is either a relic of solutionphase aggregation or an artifact of the electrospray process. The results of the (Ox-Red) $15 \alpha$-lactalbumin heterodimers seem to best support a gas-phase asymmetric charge partitioning process [56].

\section{Dissociation of Cyctochrome c Dimers}

Cytochrome $c$ homodimers form readily from electrospray solutions of high concentration $(\sim 100 \mu \mathrm{M})$ with charge states ranging from $\left[(\text { cytochrome } c)_{2}+19 \mathrm{H}\right]^{19+}$ to [(cytochrome $\left.c)_{2}+13 \mathrm{H}\right]^{13+}$ (abbreviated D19-D13). As shown previously, dissociation of cytochrome $c$ D19 formed directly from solution results in a narrow symmetric charge partitioning process [56]. MS/MS of D17 formed directly from solution results in a relatively broad but symmetric charge partitioning process (predominantly D17 $\rightarrow$ M9 + M8) (Figure 2a).

In contrast, dissociation of D13 formed directly from solution results in highly asymmetric charge partitioning centered around D13 $\rightarrow$ M9 + M4 (Figure 2c). The D17 to D19 ions were isolated and reacted with trieth- 
ylamine inside the FT-ICR ion cell for $100 \mathrm{~s}$, resulting in the formation of predominantly D13 ions. Isolating the D13 and then activating these ions results in the MS/MS spectrum shown in Figure $2 b$. The dominant process is symmetric dissociation centered around D13 $\rightarrow \mathrm{M} 7+\mathrm{M} 6$. Thus, the dissociation process of the D13 clearly depends on whether they are formed by gasphase proton transfer reduction of the D17-D19 (Figure $2 b$ ), or whether they are formed directly from solution (Figure 2c). Under these experimental conditions, the ion internal energy equilibrates with the room temperature blackbody radiation field prior to ion activation [63]. Thus, the difference in fragmentation cannot be an artifact of differences in internal energy. These results show that the structures of the D13 ions differ for these two methods of ion formation and that the dimer structure influences how these dimers dissociate, i.e., via symmetric versus asymmetric charge partitioning. It should be noted that the dimerization in solution is non-specific and that a large ensemble of dimer structures could be present. However, it is clear that at least two distinct structures or distinct ensembles of structures exist.

The "memory" of the D13 ions could be due to different dimer structures that exist in solution. These dimers may obtain different numbers of charges in the process of electrospray ionization, or the electrospray process may induce conformational changes in some fraction of the dimers upon the transition from solution into the gas phase resulting in different dimer structures for the D17 versus D13. We are not able to clearly distinguish between these two possibilities based on these results alone. However, the broad distribution in the charge states of the dimers formed from these denaturing conditions versus the typically single dominant charge state of the dimer observed by electrospray from solutions in which these molecules have a native structure suggests that the former mechanism is more likely, i.e. at least two distinct structures or ensemble of structures exist in solution and that these structures end up with different extents of charge in the electrospray process.

Similar results are observed for D15 ions formed from solution versus formed by proton transfer. Those that are formed directly from solution show a bimodal dissociation: A symmetric process centered around M8/M7 and an asymmetric process centered around M10/M5 (Figure 3a). Charge reduction of D19-D17 to form lower charge states with subsequent isolation and dissociation of D15 results in only symmetric dissociation to form M8/M7 (Figure 3b). Again, these D15 ions clearly retain a "memory" of whether they were formed in the gas phase from higher charge states that dissociate symmetrically, or directly from solution.

\section{Gas-Phase H/D Exchange of Cytochrome c Monomers and Dimers with $\mathrm{ND}_{3}$}

Previous studies showed that the extent of charge asymmetry of cytochrome $c$ D11 formed directly by

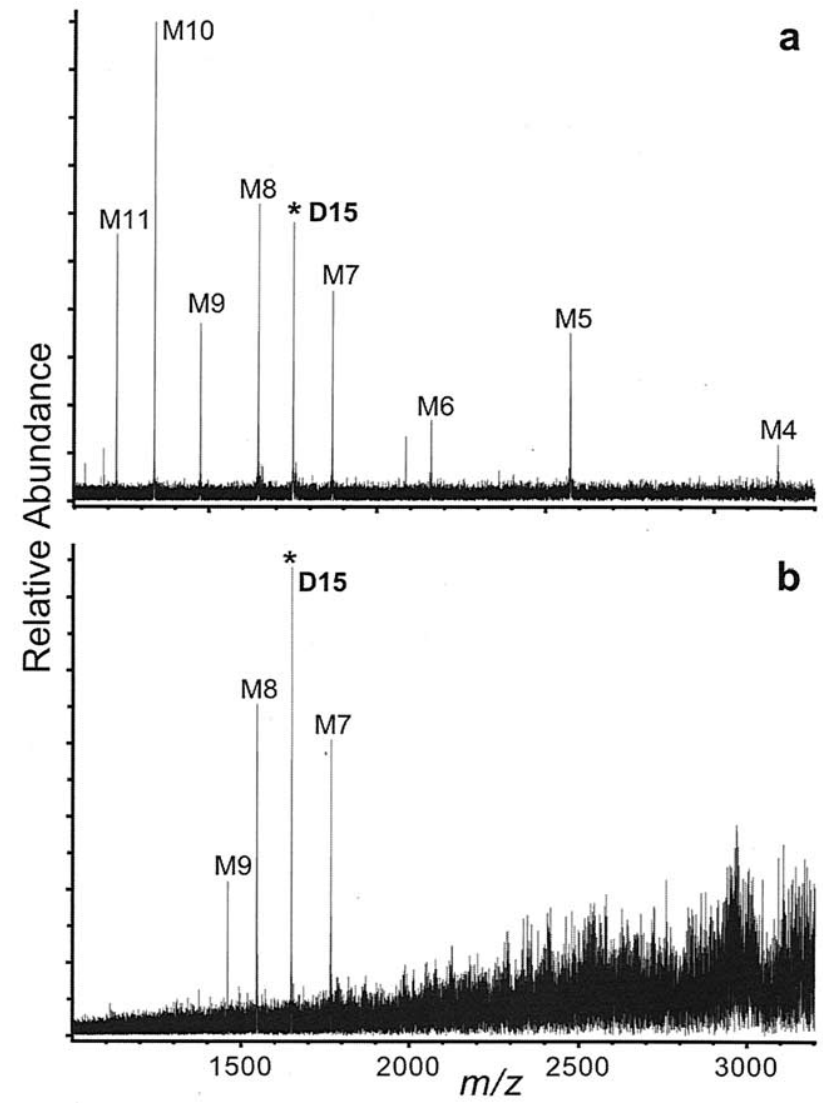

Figure 3. MS/MS spectra of (cytochrome $c$ dimers $+15 \mathrm{H})^{15+}$, (D15), formed (a) directly by ESI and (b) formed by isolation of D19-D17, gas-phase proton transfer reduction with diethylamine, isolation and subsequent activation of D15. Ions were formed from a $100 \mathrm{mM}$ solution (1:1 water:methanol $+2 \%$ acetic acid).

nanoelectrospray using aqueous solutions containing $100 \mathrm{mM}$ ammonium acetate at neutral $\mathrm{pH}$ depended on the internal energy that was deposited into the ions [56]. To try to establish the extent to which different conformers might be contributing to the observed energy dependence, $\mathrm{H} / \mathrm{D}$ exchange studies were conducted on D11. Careful adjustment of the ESI source parameters, particularly increasing the offset voltage on the external accumulation hexapole and the increasing the hexapole load time, assists in the formation of cytochrome $c$ D11 (Figure 4).

For these gas-phase $\mathrm{H} / \mathrm{D}$ exchange experiments, the pressure of $\mathrm{NH}_{3}$ was varied from $1 \times 10^{-8}$ to $3 \times 10^{-7}$ torr with a constant reaction time of $180 \mathrm{~s}$. For the experiment at the highest reagent pressure (greatest extent of $\mathrm{H} / \mathrm{D}$ exchange) (Figure 5c), the average number of hydrogens exchanged for deuterium is $\sim 170$ after subtracting the exchange of the 11 protons. The width of the distribution is approximately $110 \mathrm{Da}$. The $\mathrm{S} / \mathrm{N}$ is insufficient to attribute the variations in isotope intensities to resolvable conformers. In contrast, M7 under these same conditions shows an average exchange of $\sim 84$ hydrogen atoms for deuterium with an isotopic distribution width of $\sim 50$. Thus, both the average extent of 


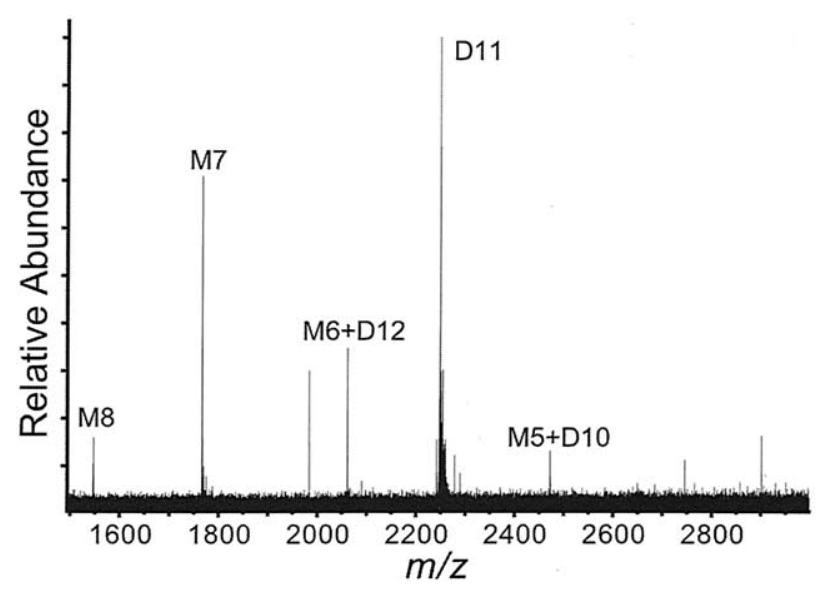

Figure 4. ESI mass spectrum of $500 \mu \mathrm{M}$ equine cytochrome $c$ in aqueous solution with $100 \mathrm{mM}$ ammonium acetate $\mathrm{pH} \sim 7$ with the spectrometer tuned to produce maximum abundance of dimers with $11+$ charges (D11).

exchange and the width of the distribution of the dimer are approximately twice that of the monomer. If only a single structure was present, the combined maximum extent of exchange and the width of the distribution would not be expected to increase linearly with ion mass, assuming that the extent of $\mathrm{H} / \mathrm{D}$ exchange is related to the exposed surface of the cluster. These
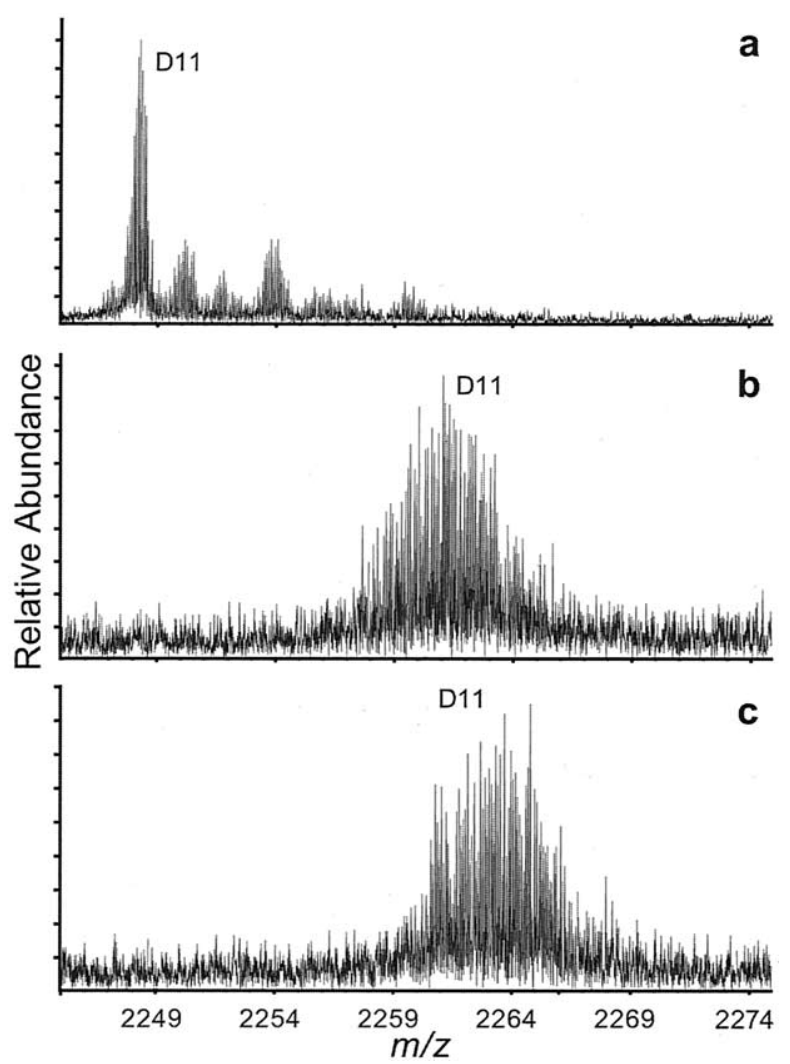

Figure 5. Partial ESI mass spectra of cytochrome $c$ (D11 region shown) using the same solution conditions as Figure 4(a) before, and after gas-phase hydrogen-deuterium exchange with $\mathrm{ND}_{3}$ for $180 \mathrm{~s}$ at (b) $1 \times 10^{-7}$ torr and (c) $3 \times 10^{-7}$ torr. results suggest the presence of multiple conformers of the dimers that are not resolved by these H/D exchange experiments, consistent with our expectation that these D11 ions are formed by nonspecific dimerization of cyctochrome $c$ molecules in solution.

Extensive gas-phase H/D exchange studies of cytochrome $c$ monomers have been conducted by McLafferty and coworkers [22-24] who discovered as many as seven conformations of cytochrome $c$ M7 formed directly by ESI [24]. In our experiments, $\mathrm{ND}_{3}$ is used as the exchange reagent instead of $\mathrm{D}_{2} \mathrm{O}$ [24], and the reaction times are shorter. The isotope distribution for the M7 suggests the presence of multiple conformers consistent with the results reported by McLafferty and coworkers.

\section{Conclusions}

The asymmetric charge partitioning that is observed upon the dissociation of protein homodimers can be explained by two different mechanisms. In one mechanism, the asymmetry is induced by the unfolding of one of the proteins in the dimer upon activation. In dimers consisting of two nearly identical proteins, the protein with the higher conformational flexibility dissociates from the complex with more charge. This can be attributed to part of the molecule exiting the dissociation barrier where further unfolding of the molecule is induced by the repulsive Coulomb potential. During this unfolding and separation process, protons are transferred to the unfolding protein to reduce overall Coulomb energy. This mechanism best explains the results observed for $\alpha$-lactabumin heterodimers consisting of one oxidized and one reduced protein. This mechanism also best explains the results for cytochrome $c$ dimers with 11 charges for which an energy dependence to the extent of asymmetry is observed [56].

Some of the observed asymmetric charge partitioning can also be attributed to a different mechanism in which the structures of the monomers in the dimer differ. This best explains the results observed for cytochrome $c$ dimers with $17+$ charges versus those with $13+$ charges. When formed directly from solution, the former dissociates with a symmetric distribution of charge whereas the latter dissociate with a highly asymmetric distribution of charge. However, when the charge state of the $17+$ dimer ions is reduced to $13+$ in the gas phase via gentle proton transfer reactions, the resulting $13+$ ions dissociate with a symmetric distribution of charge. This clearly demonstrates that the 13+ ions formed from the 17+ ions have different structures (or different ensembles of structures) than those formed directly from solution and that this structural difference is responsible for the asymmetric versus symmetric charge distributions observed upon dissociating these $13+$ ions. This is the first reported evidence that asymmetric charge partitioning can be induced by structural differences in the monomer sub-units in the complex.

Finally, H/D exchange of the 11+ charge state of cytochrome $c$ dimers indicates the presence of multiple 
conformers, although these conformers are not resolvable in this experiment. Further work with other H/D exchange reagents or different conditions, or with conformationally selective methods, such as ion mobility, in combination with dissociation experiments may provide further useful information about the charge partitioning process. A better understanding of this process will ultimately improve the structural information that can be obtained from the gas-phase dissociation of noncovalent complexes.

\section{Acknowledgments}

The authors thank Dr. David King (University of California, Berkeley) for $\alpha$-lactalbumin reduction and purification. This work has been supported by the National Institutes of Health (grant no. R01-GM64712-01).

\section{References}

1. Kaleta, D. T.; Jarrold, M. F. Peptide Pinwheels. J. Am. Chem. Soc. 2002, 124, 1154-1155.

2. Kaleta, D. T.; Jarrold, M. F. Noncovalent Interactions Between Unsolvated Peptides. J. Phys. Chem. A 2002, 106, 9655-9664.

3. Clemmer, D. E.; Jarrold, M. F. Ion Mobility Measurements and Their Applications to Clusters and Biomolecules. J. Mass Spectrom. 1997, 32, 577-592.

4. Counterman, A. E.; Valentine, S. J.; Srebalus, C. A.; Henderson, S. C.; Hoaglund, C. S.; Clemmer, D. E. High-Order Structure and Dissociation of Gaseous Peptide Aggregates That are Hidden in Mass Spectra. J. Am. Soc. Mass Spectrom. 1998, 9, 743-759.

5. Counterman, A. E.; Hilderbrand, A. E.; Barnes, C. A. S.; Clemmer, D. E. Formation of Peptide Aggregates During ESI: Size, Charge, Composition, and Contributions to Noise. J. Am. Soc. Mass Spectrom. 2001, 12, 1020-1035.

6. Gidden, J.; Wyttenbach, T.; Batka, J. J.; Weis, P.; Jackson, A. T.; Scrivens, J. H.; Bowers, M. T. Folding Energetics and Dynamics of Macromolecules in the Gas Phase: Alkali Ion-Cationized Poly(Ethylene Terephthalate) Oligomers. J. Am. Chem. Soc. 1999, 121, 1421-1422.

7. Guevremont, R.; Purves, R. W. High Field Asymmetric Waveform Ion Mobility Spectrometry-Mass Spectrometry: An Investigation of Leucine Enkephalin Ions Produced by Electrospray Ionization. J. Am. Soc. Mass Spectrom. 1999, 10, 492-501.

8. Purves, R. W.; Barnett, D. A.; Guevremont, R. Separation of Protein Conformers Using Electrospray-High Field Asymmetric Waveform Ion Mobility Spectrometry-Mass Spectrometry. Int. J. Mass Spectrom. 2000, 197, 163-177.

9. Deng, Y. Z.; Zhang, Z. Q.; Smith, D. L. Comparison of Continuous and Pulsed Labeling Amide Hydrogen Exchange/Mass Spectrometry for Studies of Protein Dynamics. J. Am. Soc. Mass Spectrom. 1999, 10, 675-684.

10. Zhu, M. M.; Rempel, D. L.; Gross, M. L. Modeling Data from Titration, Amide H/D Exchange, and Mass Spectrometry to Obtain Protein-Ligand Binding Constants. J. Am. Soc. Mass Spectrom. 2004, 15, 388-397.

11. Gard, E.; Willard, D.; Bregar, J.; Green, M. K.; Lebrilla, C. B. Site-Specificity in the H-D Exchange-Reactions of Gas-Phase Protonated Amino-Acids with CH3OD. Org. Mass Spectrom. 1993, 28, 1632-1639.

12. Campbell, S.; Rodgers, M. T.; Marzluff, E. M.; Beauchamp, J. L. Structural and Energetic Constraints on Gas-Phase HydrogenDeuterium Exchange Reactions of Protonated Peptides with D2O, CD3OD, CD3CO2D, and ND3. J. Am. Chem. Soc. 1994, $116,9765-9766$.
13. Gard, E.; Green, M. K.; Bregar, J.; Lebrilla, C. B. Gas-Phase Hydrogen-Deuterium Exchange as a Molecular Probe for the Interaction of Methanol and Protonated Peptides. J. Am. Soc. Mass Spectrom. 1994, 5, 623-631.

14. Campbell, S.; Rodgers, M. T.; Marzluff, E. M.; Beauchamp, J. L. Deuterium Exchange Reactions as a Probe of Biomolecule Structure. Fundamental Studies of Gas Phase H/D Exchange Reactions of Protonated Glycine Oligomers with D2O, CD3OD, CD3CO2D, and ND3. J. Am. Chem. Soc. 1995, 117, 12840-12854.

15. Green, M. K.; Penn, S. G.; Lebrilla, C. B. The Complexation of Protonated Peptides with Saccharides in the Gas Phase Decreases the Rates of Hydrogen/Deuterium Exchange Reactions. J. Am. Soc. Mass Spectrom. 1995, 6, 1247-1251.

16. Kaltashov, I. A.; Doroshenko, V. M.; Cotter, R. J. Gas Phase Hydrogen/Deuterium Exchange Reactions of Peptide Ions in a Quadrupole Ion Trap Mass Spectrometer. Proteins 1997, 28 , 53-58.

17. Heck, A. J. R.; Jorgensen, T. J. D.; O'Sullivan, M.; von Raumer, M.; Derrick, P. J. Gas-Phase Noncovalent Interactions Between Vancomycin-Group Antibiotics and Bacterial Cell-Wall Precursor Peptides Probed by Hydrogen/Deuterium Exchange. J. Am. Soc. Mass Spectrom. 1998, 9, 1255-1266.

18. Freitas, M. A.; Marshall, A. G. Rate and Extent of Gas-Phase Hydrogen/Deuterium Exchange of Bradykinins: Evidence for Peptide Zwitterions in the Gas Phase. Int. J. Mass Spectrom. 1999, 183, 221-231.

19. Reyzer, M. L.; Brodbelt, J. S. Gas-Phase H/D Exchange Reactions of Polyamine Complexes: $[\mathrm{M}+\mathrm{H})(+)$, (M plus alkali metal $(+)]$, and $(\mathrm{M}+2 \mathrm{H})(2+)$. J. Am. Soc. Mass Spectrom. 2000, 11, 711-721.

20. Levy-Seri, E.; Koster, G.; Kogan, A.; Gutman, K.; Reuben, B. G.; Lifshitz, C. An Electrospray Ionization-Flow Tube Study of H/D Exchange in Protonated Bradykinin. J. Phys. Chem. A 2001, 105, 5552-5559.

21. Solouki, T.; Fort, R. C.; Alomary, A.; Fattahi, A. Gas Phase Hydrogen-Deuterium Exchange Reactions of a Model Peptide: FT-ICR and Computational Analyses of Metal Induced Conformational Mutations. J. Am. Soc. Mass Spectrom. 2001, 12, 1272-1285.

22. Suckau, D.; Shi, Y.; Beu, S. C.; Senko, M. W.; Quinn, J. P.; Wampler, F. M.; McLafferty, F. W. Coexisting Stable Conformations of Gaseous Protein Ions. Proc. Nat. Acad. Sci. U.S.A. 1993, 90, 790-793.

23. Wood, T. D.; Chorush, R. A.; Wampler, F. M.; Little, D. P.; O'Connor, P. B.; McLafferty, F. W. Gas-Phase Folding and Unfolding of Cytochrome-c Cations. Proc. Natl. Acad. Sci. U.S.A. 1995, 92, 2451-2454.

24. McLafferty, F. W.; Guan, Z. Q.; Haupts, U.; Wood, T. D.; Kelleher, N. L. Gaseous Conformational Structures of Cytochrome c. J. Am. Chem. Soc. 1998, 120, 4732-4740.

25. Reid, G. E.; O’Hair, R. A. J.; Styles, M. L.; McFadyen, W. D.; Simpson, R. J. Gas Phase Ion-Molecule Reactions in a Modified Ion Trap: H/D Exchange of Noncovalent Complexes and Coordinatively Unsaturated Platinum Complexes. Rapid Commun. Mass Spectrom. 1998, 12, 1701-1708.

26. Kogan, A.; Ustyuzhanin, P.; Reuben, B. G.; Lifshitz, C. Hydrogen/Deuterium Exchange of Monomers and Dimers of Leucine Enkephalin. Int. J. Mass Spectrom. 2002, 213, 1-4.

27. Mao, D.; Douglas, D. J. H/D Exchange of Gas Phase Bradykinin Ions in a Linear Quadrupole Ion Trap. J. Am. Soc. Mass Spectrom. 2003, 14, 85-94.

28. Lee, S. W.; Lee, H. N.; Kim, H. S.; Beauchamp, J. L. Selective Binding of Crown Ethers to Protonated Peptides Can be Used to Probe Mechanisms of H/D Exchange and Collision-Induced Dissociation Reactions in the Gas Phase. J. Am. Chem. Soc. 1998, 120, 5800-5805. 
29. Gross, D. S.; Schnier, P. D.; Rodriguezcruz, S. E.; Fagerquist, C. K.; Williams, E. R. Conformations and Folding of Lysozyme Ions in Vacuo. Proc. Nat. Acad. Sci. U.S.A. 1996, 93, 3143-3148.

30. Williams, E. R. Proton Transfer Reactivity of Large Multiply Charged Ions. J. Mass Spectrom. 1996, 31, 831-842.

31. Loo, J. A. Studying Noncovalent Protein Complexes by Electrospray Ionization Mass Spectrometry. Mass Spectrom. Rev. 1997, 16, 1-23.

32. Lim, H. K.; Hsieh, Y. L.; Ganem, B.; Henion, J. Recognition of Cell-Wall Peptide Ligands by Vancomycin Group Antibiotics-Studies Using Ion-Spray Mass-Spectrometry. J. Mass Spectrom. 1995, 30, 708-714.

33. Loo, J. A.; Hu, P. F.; McConnell, P.; Mueller, W. T.; Sawyer, T. K.; Thanabal, V. A Study of Src SH2 Domain ProteinPhosphopeptide Binding Interactions by Electrospray Ionization Mass Spectrometry. J. Am. Soc. Mass Spectrom. 1997, 8, 234-243.

34. Jorgensen, T. J. D.; Roepstorff, P.; Heck, A. J. R. Direct Determination of Solution Binding Constants for Noncovalent Complexes Between Bacterial Cell Wall Peptide Analogues and Vancomycin Group Antibiotics by Electrospray Ionization Mass Spectrometry. Anal. Chem. 1998, 70, 4427-4432.

35. Daniel, J. M.; Friess, S. D.; Rajagopalan, S.; Wendt, S.; Zenobi, R. Quantitative Determination of Noncovalent Binding Interactions Using Soft Ionization Mass Spectrometry. Int. J. Mass Spectrom. 2002, 216, 1-27.

36. Gross, D. S.; Zhao, Y. X.; Williams, E. R. Dissociation of Heme-Globin Complexes by Blackbody Infrared Radiative Dissociation: Molecular Specificity in the Gas Phase? J. Am. Soc. Mass Spectrom. 1997, 8, 519-524.

37. Schnier, P. D.; Klassen, J. S.; Strittmatter, E. E.; Williams, E. R. Activation Energies for Dissociation of Double Strand Oligonucleotide Anions: Evidence for Watson-Crick Base Pairing in Vacuo. J. Am. Chem. Soc. 1998, 120, 9605-9613.

38. Rogalewicz, F.; Hoppilliard, Y.; Ohanessian, G. Structures and Fragmentations of Zinc(II) Complexes of Amino Acids in the Gas Phase. IV. Solvent Effect on the Structure of Electrosprayed Ions. Int. J. Mass Spectrom. 2003, 227, 439-451.

39. Gabelica, V.; De Pauw, E. Comparison Between SolutionPhase Stability and Gas-Phase Kinetic Stability of Oligodeoxynucleotide Duplexes. J. Mass Spectrom. 2001, 36, 397-402.

40. Gabelica, V.; De Pauw, E. Comparison of the CollisionInduced Dissociation of Duplex DNA at Different Collision Regimes: Evidence for a Multistep Dissociation Mechanism. J. Am. Soc. Mass Spectrom. 2002, 13, 91-98.

41. Gabelica, V.; De Pauw, E. Collision-Induced Dissociation of 16-mer DNA Duplexes with Various Sequences: Evidence for Conservation of the Double Helix Conformation in the Gas Phase. Int. J. Mass Spectrom. 2002, 219, 151-159.

42. Wan, K. X.; Shibue, T.; Gross, M. L. Noncovalent Complexes Between DNA-Binding Drugs and Double-Stranded Oligodeoxynucleotides: A Study by ESI Ion-Trap Mass Spectrometry. J. Am. Chem. Soc. 2000, 122, 300-307.

43. Chen, Y. L.; Campbell, J. M.; Collings, B. A.; Konermann, L.; Douglas, D. J. Stability of a Highly Charged Noncovalent Complex in the Gas Phase: Holomyoglobin. Rapid Commun. Mass Spectrom. 1998, 12, 1003-1010.

44. Hunter, C. L.; Mauk, A. G.; Douglas, D. J. Dissociation of Heme from Myoglobin and Cytochrome $b(5)$ : Comparison of Behavior in Solution and the Gas Phase. Biochem. U.S.A. 1997, 36, 1018-1025.

45. Jorgensen, T. J. D.; Delforge, D.; Remacle, J.; Bojesen, G.; Roepstorff, P. Collision-Induced Dissociation of Noncovalent Complexes Between Vancomycin Antibiotics and Peptide Ligand Stereoisomers: Evidence for Molecular Recognition in the Gas Phase. Int. J. Mass Spectrom. 1999, 188, 63-85.
46. Loo, J. A.; He, J. X.; Cody, W. L. Higher Order Structure in the Gas Phase Reflects Solution Structure. J. Am. Chem. Soc. 1998, 120, 4542-4543.

47. Rostom, A. A.; Fucini, P.; Benjamin, D. R.; Juenemann, R.; Nierhaus, K. H.; Hartl, F. U.; Dobson, C. M.; Robinson, C. V. Detection and Selective Dissociation of Intact Ribosomes in a Mass Spectrometer. Proc. Nat. Acad. Sci. U.S.A. 2000, 97, 5185-5190.

48. Pinkse, M. W. H.; Maier, C. S.; Kim, J. I.; Oh, B. H.; Heck, A. J. R. Macromolecular Asserably of Helicobacter pylori Urease Investigated by Mass Spectrometry. J. Mass Spectrom. 2003, 38, 315-320.

49. Light-Wahl, K. J.; Schwartz, B. L.; Smith, R. D. Observation of the Noncovalent Quaternary Associations of Proteins by Electrospray Ionization Mass Spectrometry. J. Am. Chem. Soc. 1994, 116, 5271-5278.

50. Schwartz, B. L.; Bruce, J. E.; Anderson, G. A.; Hofstadler, S. A.; Rockwood, A. L.; Smith, R. D.; Chilkoti, A.; Stayton, P. S. Dissociation of Tetrameric Ions of Noncovalent Streptavidin Complexes Formed By Electrospray Ionization. J. Am. Soc. Mass Spectrom. 1995, 6, 459-465.

51. Fitzgerald, M. C.; Chernushevich, I.; Standing, K. G.; Whitman, C. P.; Kent, S. B. H. Probing the Oligomeric Structure of an Enzyme By Electrospray Ionization Time-of-Flight Mass Spectrometry. Proc. Nat. Acad. Sci. U.S.A. 1996, 93, 6851-6856.

52. Felitsyn, N.; Kitova, E. N.; Klassen, J. S. Thermal Decomposition of a Gaseous Multiprotein Complex Studied by Blackbody Infrared Radiative Dissociation. Investigating the Origin of the Asymmetric Dissociation Behavior. Anal. Chem. 2001, 73, 4647-4661.

53. Benesch, J. L. P.; Sobott, F.; Robinson, C. V. Thermal Dissociation of Multimeric Protein Complexes by Using Nanoelectrospray Mass Spectrometry. Anal. Chem. 2003, 75, 2208-2214.

54. http://www.rcsb.org/pdb/ entry 2IZA.

55. Versluis, C.; van der Staaij, A.; Stokvis, E.; Heck, A. J. R.; de Craene, B. Metastable Ion Formation and Disparate Charge Separation in the Gas-Phase Dissection of Protein Assemblies Studied by Orthogonal Time-of-Flight Mass Spectrometry. J. Am. Soc. Mass Spectrom. 2001, 12, 329-336.

56. Jurchen, J. C.; Williams, E. R. Origin of Asymmetric Charge Partitioning in the Dissociation of Gas-Phase Protein Homodimers. J. Am. Chem. Soc. 2003, 125, 2817-2826.

57. Jurchen, J. C.; Garcia, D. E.; Williams, E. R. Gas-Phase Dissociation Pathways of Multiply Charged Peptide Clusters. J. Am. Soc. Mass Spectrom. 2003, 14, 1373-1386.

58. Gauthier, J. W.; Trautman, T. R.; Jacobson, D. B. Sustained Off-Resonance Irradiation for Collision-Activated Dissociation Involving Fourier-Transform Mass-Spectrometry-CollisionActivated Dissociation Technique That Emulates Infrared Multiphoton Dissociation. Anal. Chim. Acta 1991, 246, 211- 225.

59. Winger, B. E.; Light-Wahl, K. J.; Rockwood, A. L.; Smith, R. D. Probing Qualitative Conformation Differences of Multiply Protonated Gas-Phase Proteins via H/D Isotopic Exchange With D2O. J. Am. Chem. Soc. 1992, 114, 5897-5898.

60. Felitsyn, N.; Kitova, E. N.; Klassen, J. S. Thermal Dissociation of the Protein Homodimer Ecotin in the Gas Phase. J. Am. Soc. Mass Spectrom. 2002, 13, 1432-1442.

61. Apostol, I. Assessing the Relative Stabilities of Engineered Hemoglobins Using Electrospray Mass Spectrometry Anal. Biochem. 1999, 272, 8-18.

62. Versluis, C.; Heck, A. J. R. Gas-Phase Dissociation of Hemoglobin. Int. J. Mass Spectrom. 2001, 210, 637-649.

63. Price, W. D.; Williams, E. R. Activation of Peptide Ions by Blackbody Radiation: Factors That Lead to Dissociation Kinetics in the Rapid Energy Exchange Limit. J. Phys. Chem. A 1997, 101, 8844-8852. 